\title{
A Novel Efficient Technique for the Calculation of the Green's Functions in Rectangular Waveguides Based on Accelerated Series Decomposition
}

\author{
Francisco Javier Perez Soler, Student Member, IEEE, Fenando D. Quesada Pereira, Member, IEEE, \\ David Cañete Rebenaque, Student Member, IEEE, Alejandro Alvarez Melcon, Member, IEEE, and \\ Juan R. Mosig, Fellow, IEEE
}

\begin{abstract}
A new efficient technique for computing the Green's functions inside rectangular waveguides is presented. After a summary of the classical approaches and their difficulties, a new strategy is proposed, based on the decomposition of the main spectral series into simpler terms. Although the resulting series present better convergence rate, several acceleration techniques are combined to further improve the efficiency. Several results are presented to demonstrate the improvements in convergence rates obtained using the new decomposition.
\end{abstract}

Index Terms-Acceleration techniques, dyadic Green's functions, Ewald methods, integral equations, rectangular waveguide.

\section{INTRODUCTION}

$\mathbf{T}$ HE calculation of the Green's functions inside waveguides and cavities has been an interesting subject largely investigated in the last decades [1]-[4]. This is because the important role that these Green's functions play during the analysis and design of shielded microwave circuits (MMIC) and waveguide devices, using the integral equation technique [5]-[7].

In particular, the Green's functions inside rectangular waveguides have well-known expressions consisting on infinite series, either in the spectral or in the spatial domains [8]-[10]. However, despite their analytical simplicity, these series present some inconveniences from the computational point of view, as they exhibit very slow convergence rates. As a result, the number of terms in the series (modes of the cavity or spatial images), needed to obtain accurate results, is in general very high. This leads to high computational times when computing several hundreds of Green's functions, needed during the analysis of practical devices using Integral Equation formulations. These are the main reasons for the interest in finding methods that allow to accelerate the convergence of the relevant series.

Recently, several approaches based on the Ewald method [1], [11]-[13] for the series acceleration have been introduced. With the Ewald technique it is possible to achieve excellent convergence rates employing only a small number of terms in the series. The key of this method consist on the precise choice of a so

Manuscript received December 4, 2007. Current version published October 3, 2008.

F. J. P. Soler, F. D. Q. Pereira, D. C. Rebenaque, and A. A. Melcon are with the Technical University of Cartagena, E-30202 Cartagena, Spain.

J. R. Mosig is with the Swiss Federal Institute of Technology, LEMA-EPFL, Lausanne CH-1015, Switzerland.

Digital Object Identifier 10.1109/TAP.2008.929438

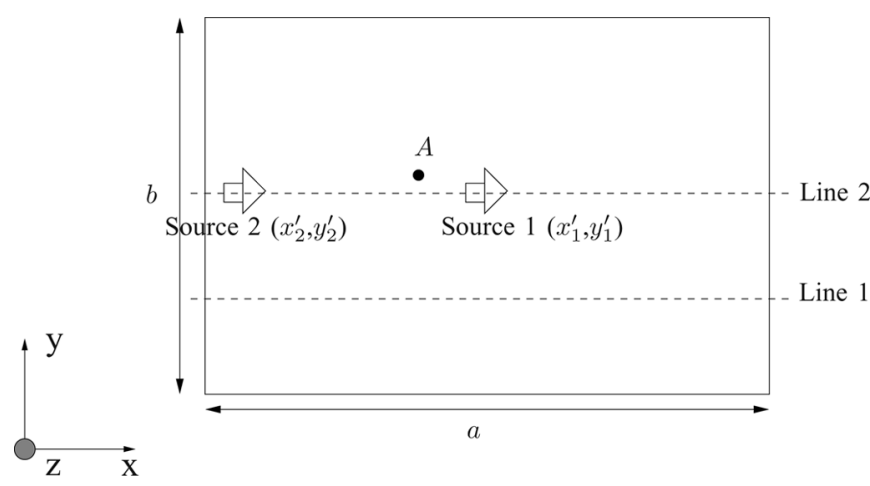

Fig. 1. Geometry under study. It consists of a cross-sectional plane $\left(z=z^{\prime}\right)$ of an infinite rectangular waveguide on which different source and observation points are placed. Point $A$ has been set to $(x=0.35 a, y=0.52 b)$, and will be used for convergence studies later in the theory section. Lines 1 and 2 will be employed for spatial sweeps during the validation in the results section, and have been set to $(y=0.24 b)$ and $(y=0.5 b)$, respectively. The two sources have been located at $\left(x_{1}^{\prime}=0.5 a, y_{1}^{\prime}=0.5 b\right)$ and $\left(x_{2}^{\prime}=0.1 a, y_{1}^{\prime}=0.5 b\right)$.

called splitting parameter $(E)$, which is critical for the improvement of the convergence, and for the numerical stability of the algorithm [2], [13], [14]. Besides, the method employs complementary error functions (Erfc) of complex arguments in the calculations which, as a counterpart, are known to be a bottleneck for computational cost. On the other hand, alternative methods were derived in the past for the acceleration of the spectral series [5], [10]. While these methods are also efficient, they usually fail close to the singularity presented by the source, where a large number of terms must be included before the technique starts to be effective. In this paper, after a brief review of the previously mentioned approaches, we present a novel strategy for the calculation of the Green's functions inside rectangular waveguides (basic geometry is shown in Fig. 1).

The technique splits the original spectral series into several terms, obtained through a Taylor series expansion. The Kummer technique [2] is then applied using these new computed terms, to improve the convergence of the remaining series. The final step is how to evaluate the individual Taylor terms. For the first order term, the Ewald transformation is used. Because of the nature of this first order term, the Ewald transformation has shown to be extremely efficient in this case. First, since the term is static there are no problems in the selection of the splitting parameter. Also, the complementary error function now becomes of real arguments, allowing faster calculations. 
In addition, the higher order Taylor terms already converge faster. Moreover, due to the static nature of these terms they are computed only once in a given frequency range. This fact can accelerate considerably the calculation of the Green's functions during the analysis of practical circuits inside a given bandwidth. Applying the Kummer technique with these terms, a fast convergent dynamic series is obtained. Only this dynamic series needs to be evaluated for each frequency point. As a further acceleration method, the summation by parts technique proposed in [10] is used when the observation point is far from the source point. There is an important difference in the application of the summation by parts technique, with respect to the work presented in [9]. In [9], the summation by parts technique is applied sequentially to the two dimensional series. This restricts the use of the technique to observer points far from the source in the two coordinates $(x, y)$ of the cross sectional plane (see Fig. 1). On the contrary, in this work we apply the summation by parts technique only to the inner series of the Green's functions. In this work it is shown that, once the inner series is accurately computed with the summation by parts technique, the outer series exhibits a very fast convergence behavior. If the observation point has the $(y)$ coordinate close to the source $\left(y^{\prime}\right)$, the inner and outer series are swapped, and the summation by parts technique is applied along the $x$-axis. By introducing this algorithm, the range of applicability of the summation by parts technique is extended, since now all observation points can be accelerated, except those situated very close the source point along the two directions $(x, y)$ of the cross section.

In this paper we show the big improvement in efficiency that is obtained when using the new strategy proposed for the calculation of the rectangular waveguide Green's functions. Comparisons of convergence rates using the different methods proposed are included. We also present results that validate the new techniques, and show the advantages of the proposed strategy in terms of computational time.

\section{TheoreticAl OUtLINE}

\section{A. Revision of the Different Approaches}

The well-known expressions for the three components of the magnetic potential dyadic Green's function $\overline{\bar{G}}_{A}$, in the spectral domain, are [12]

$$
\begin{aligned}
G A_{x x}= & \frac{\mu}{2 a b} \sum_{m, n=0}^{\infty} V_{m, n} \cos \left(\frac{m \pi x}{b}\right) \cos \left(\frac{m \pi x^{\prime}}{a}\right) \\
& \times \sin \left(\frac{n \pi y}{b}\right) \sin \left(\frac{n \pi y^{\prime}}{b}\right) \\
G A_{y y}= & \frac{\mu}{2 a b} \sum_{m, n=0}^{\infty} V_{m, n} \sin \left(\frac{m \pi x}{b}\right) \sin \left(\frac{m \pi x^{\prime}}{a}\right) \\
& \times \cos \left(\frac{n \pi y}{b}\right) \cos \left(\frac{n \pi y^{\prime}}{b}\right) \\
G A_{z z}= & \frac{\mu}{2 a b} \sum_{m, n=0}^{\infty} V_{m, n} \sin \left(\frac{m \pi x}{b}\right) \sin \left(\frac{m \pi x^{\prime}}{a}\right) \\
& \times \sin \left(\frac{n \pi y}{b}\right) \sin \left(\frac{n \pi y^{\prime}}{b}\right)
\end{aligned}
$$

where we have used the following definitions:

$$
\begin{aligned}
V_{m, n} & =\frac{\epsilon_{m} \epsilon_{n}}{\gamma_{m, n}} e^{-\gamma_{m, n}\left|z-z^{\prime}\right|} ; \quad \epsilon_{i}= \begin{cases}1 & i=0 \\
2 & i \neq 0\end{cases} \\
\gamma_{m, n} & =\sqrt{k_{\rho_{m, n}}^{2}-k^{2}} \\
k_{\rho_{m, n}}^{2} & =\left(\frac{m \pi}{a}\right)^{2}+\left(\frac{n \pi}{b}\right)^{2}
\end{aligned}
$$

and $(a, b)$ are the dimensions of the rectangular waveguide (see Fig. 1). Also, $(k)$ is the propagation constant of the homogeneous medium filling the waveguide.

The computation of this series can be performed easily, and a rapid convergence is achieved for large values of $\left|z-z^{\prime}\right|$. Unfortunately, when the difference of the $z$-coordinates is close to zero, the number of terms needed to achieve convergence rapidly increases, until the series become non-convergent in the limiting $\left(z=z^{\prime}\right)$ case (when source and observation points are on the same cross-sectional plane; for instance point $A$ in Fig. 1).

On the other hand, the same components can be written in the spatial domain, through the use of the Poisson summation formula applied to the previous expressions. This yields to the following alternative series [12]:

$$
\begin{aligned}
G A_{\kappa \kappa} & =\frac{\mu}{4 \pi} \sum_{m, n=-\infty}^{\infty} \sum_{i=0}^{3} A_{i}^{\kappa \kappa} \frac{e^{-j k R_{i, m n}}}{R_{i, m n}} \\
\kappa & =x, y, z \\
R_{i, m n} & =\sqrt{\left(X_{i}+2 m a\right)^{2}+\left(Y_{i}+2 n b\right)^{2}+\left(z-z^{\prime}\right)^{2}} \\
X_{i} & = \begin{cases}x-x^{\prime} ; & i=0,1 \\
x+x^{\prime} ; & i=2,3\end{cases} \\
Y_{i} & = \begin{cases}y-y^{\prime} ; & i=0,2 \\
y+y^{\prime} ; & i=1,3\end{cases}
\end{aligned}
$$

where the only difference for the three components are the signs of the corresponding term $A_{i}^{\kappa \kappa}$, namely

$$
\begin{aligned}
& A_{i}^{x x}= \begin{cases}+1 ; & i=0,2 \\
-1 ; & i=1,3\end{cases} \\
& A_{i}^{y y}= \begin{cases}+1 ; & i=0,1 \\
-1 ; & i=2,3\end{cases} \\
& A_{i}^{z z}= \begin{cases}+1 ; & i=0,3 \\
-1 ; & i=1,2 .\end{cases}
\end{aligned}
$$

These spatial series have also a major drawback, as they converge very slowly, being necessary many terms to achieve acceptable accuracy.

In order to reduce the number of terms needed to compute the Green's functions for this kind of problems, a solution was proposed in [12]. In that work the Ewald method [15] was applied to the spatial domain series, obtaining a final decomposition in two terms. The first one is evaluated in the spectral domain as

$$
\begin{aligned}
G A_{x x 1}= & \frac{\mu}{4 a b} \sum_{m, n=0}^{\infty} \frac{\epsilon_{m} \epsilon_{n}}{\gamma_{m, n}} f\left(\left|z-z^{\prime}\right|, \gamma_{m, n}, E\right) \\
& \times \cos \left(\frac{m \pi x}{b}\right) \cos \left(\frac{m \pi x^{\prime}}{a}\right) \\
& \times \sin \left(\frac{n \pi y}{b}\right) \sin \left(\frac{n \pi y^{\prime}}{b}\right)
\end{aligned}
$$




$$
\begin{aligned}
G A_{y y 1}= & \frac{\mu}{4 a b} \sum_{m, n=0}^{\infty} \frac{\epsilon_{m} \epsilon_{n}}{\gamma_{m, n}} f\left(\left|z-z^{\prime}\right|, \gamma_{m, n}, E\right) \\
& \times \sin \left(\frac{m \pi x}{b}\right) \sin \left(\frac{m \pi x^{\prime}}{a}\right) \\
& \times \cos \left(\frac{n \pi y}{b}\right) \cos \left(\frac{n \pi y^{\prime}}{b}\right) \\
G A_{z z 1}= & \frac{\mu}{4 a b} \sum_{m, n=0}^{\infty} \frac{\epsilon_{m} \epsilon_{n}}{\gamma_{m, n}} f\left(\left|z-z^{\prime}\right|, \gamma_{m, n}, E\right) \\
& \times \sin \left(\frac{m \pi x}{b}\right) \sin \left(\frac{m \pi x^{\prime}}{a}\right) \\
& \times \sin \left(\frac{n \pi y}{b}\right) \sin \left(\frac{n \pi y^{\prime}}{b}\right) \\
f\left(\left|z-z^{\prime}\right|, \gamma_{m, n}, E\right)= & \exp \left(+\gamma_{m, n}\left(z-z^{\prime}\right)\right) \\
& \times \operatorname{Erfc}\left(\frac{\gamma_{m, n}}{2 E+\left(z-z^{\prime}\right) E}\right) \\
& +\exp \left(-\gamma_{m, n}\left(z-z^{\prime}\right)\right) \\
& \times \operatorname{Erfc}\left(\frac{\gamma_{m, n}}{2 E-\left(z-z^{\prime}\right) E}\right)
\end{aligned}
$$

while the second term is formulated in the spatial domain as

$$
\begin{aligned}
G A_{\kappa \kappa}= & \frac{\mu}{4 \pi} \sum_{m, n=-\infty}^{\infty} \sum_{i=0}^{3} A_{i}^{\kappa \kappa} \frac{1}{R_{i, m n}} \\
\times & {\left[\exp \left(-j k R_{i, m n}\right) \operatorname{Erfc}\left(\frac{R_{i, m n} E-j k}{2 E}\right)\right.} \\
& \left.+\exp \left(j k R_{i, m n}\right) \operatorname{Erfc}\left(\frac{R_{i, m n} E+j k}{2 E}\right)\right]
\end{aligned}
$$

The total Green's function will be the sum of these two terms for each component of the dyadic. It can be noticed that this method is a combination of the spectral and spatial approaches, both weighted by the use of complementary error functions, which are controlled by means of the so called splitting parameter $(E)$. If the value of the parameter is wisely chosen, these series converge with very few terms, including the case of $\left(z=z^{\prime}\right)$. Thus, the Ewald method becomes a much better option than a straight direct summation of the series. Unfortunately, precisely because of the presence of complementary error functions, this method has two main disadvantages. The first one is that this kind of functions have large computational costs, specially in the case of complex arguments. Note that complex arguments appear in (5), and also in (6) when $\gamma_{m, n}$ becomes imaginary (which occurs with the propagating modes of the waveguide).

In order to overcome this problem, in [12] an alternative expression for the case of lossless waveguides was given. The expression was based on the Taylor series expansion of the (Erfc) function, allowing the calculation of new error functions with real arguments. The second drawback of this formulation is the difficulty in selecting appropriately the splitting parameter $(E)$ for all values of the complementary error function arguments. This parameter strongly affects the convergence rate of the series, but also the numerical accuracy of the complementary error function. An incorrect selection of the splitting parameter will lead to either slow convergence rate of the series, or serious numerical errors. The numerical errors are due to the evaluation of complementary error functions for arguments with very large imaginary part. There are some studies proposing criteria for the correct choice of the optimum value of the splitting parameter [1], [13], [14], which, for these kinds of cavities, it has been found to be

$$
E_{\text {opt }}=\sqrt{\frac{\pi}{a b}} .
$$

Nevertheless, it has been observed that when the electrical size of the waveguide increases, the value of $(E)$ must be even larger than the proposed optimum in (8). In [13] another alternative criterion is proposed, which takes into account the effect of the frequency (electrical size of the cavity)

$$
E_{\mathrm{opt}, 2}=\frac{k_{z 0}}{2 \sqrt{H^{2}}}
$$

being $k_{z 0}=\left|\gamma_{10}\right|$ the propagation constant of the first allowed mode, and $H^{2}$ the largest argument allowed, without lost of precision, in the (Erfc) function. Then, the value of $(E)$ is selected as the maximum between $\left(E_{\mathrm{opt}}, E_{\mathrm{opt}, 2}\right)$. When $(E)$ increases, so does the number of terms needed to achieve convergence, resulting into a greater computational cost.

In Section II-B we will introduce an alternative technique that takes profit of the advantages of the Kummer and Ewald approaches, avoiding the main difficulties cited above.

\section{B. Decomposition in Dynamic and Static Series in the Spectral Domain}

We will start again from the modal series in the spectral domain shown in (1). The Kummer technique is based on considering the asymptotic term of the series. In this situation the expression of $k_{\rho_{m, n}}^{2}$ will be much larger than $\left(k^{2}\right)$, so we will have: $\gamma_{m, n} \approx k_{\rho_{m, n}}^{2}$. Taking for example the expression of the $G A_{z z}$ component, we write

$$
\begin{aligned}
G A_{z z}^{(a s y)}=\frac{\mu}{2 a b} & \sum_{m, n=0}^{\infty} V_{m, n}^{(a s y)} \sin \left(\frac{m \pi x}{b}\right) \\
& \times \sin \left(\frac{m \pi x^{\prime}}{a}\right) \sin \left(\frac{n \pi y}{b}\right) \sin \left(\frac{n \pi y^{\prime}}{b}\right)
\end{aligned}
$$

where we have defined the asymptotic term as

$$
V_{m, n}^{(a s y)}=\frac{\epsilon_{m} \epsilon_{n}}{k_{\rho_{m, n}}} e^{-k_{\rho m, n}\left|z-z^{\prime}\right|} .
$$

Now, if we add and subtract this asymptotic term to the original series, we obtain

$$
\begin{aligned}
G A_{z z}= & G A_{z z}-G A_{z z}^{(a s y)}+G A_{z z}^{(a s y)} \\
= & \frac{\mu}{2 a b} \sum_{m, n=0}^{\infty}\left(V_{m, n}-V_{m, n}^{(a s y)}\right) \\
& \times \sin \left(\frac{m \pi x}{b}\right) \sin \left(\frac{m \pi x^{\prime}}{a}\right) \sin \left(\frac{n \pi y}{b}\right) \sin \left(\frac{n \pi y^{\prime}}{b}\right) \\
& +\frac{\mu}{2 a b} \sum_{m, n=0}^{\infty} V_{m, n}^{a s y} \\
& \times \sin \left(\frac{m \pi x}{b}\right) \sin \left(\frac{m \pi x^{\prime}}{a}\right) \sin \left(\frac{n \pi y}{b}\right) \sin \left(\frac{n \pi y^{\prime}}{b}\right) \\
= & G A_{z z}^{\text {dynamic }}+G A_{z z}^{\text {static }} .
\end{aligned}
$$


The two series $G A_{z z}^{\text {dynamic }}$ and $G A_{z z}^{\text {static }}$ are the so called dynamic and static parts of the original series, respectively. In the dynamic part, as indexes $(m, n)$ increase, the term $V_{m, n}$ will tend to $V_{m, n}^{(a s y)}$, so the difference of the two terms will tend to zero. This will increase the convergence rate of this dynamic series. In addition, the problem of the divergence of the series in $\left(z=z^{\prime}\right)$ is solved for this term, since the limiting condition $\left(V_{m, n} \rightarrow V_{m, n}^{(a s y)}\right)$ will be true irrespective of the source and observer points positions.

What remains, then, is the calculation of the asymptotic term shown in (12), in an efficient way. The key point of the proposed technique is to apply the Ewald method to this asymptotic term. It is important to notice that the resulting expressions are very similar to those presented in equations (6) and (5). However, an important difference arises in this case. In fact, in the limiting case the following simplified expressions is obtained:

$$
\begin{aligned}
G A_{x x 1}= & \frac{\mu}{4 a b} \sum_{m, n=0}^{\infty} \frac{\epsilon_{m} \epsilon_{n}}{k_{\rho_{m, n}}} f\left(\left|z-z^{\prime}\right|, k_{\rho_{m, n}}, E\right) \\
& \times \cos \left(\frac{m \pi x}{b}\right) \cos \left(\frac{m \pi x^{\prime}}{a}\right) \\
& \times \sin \left(\frac{n \pi y}{b}\right) \sin \left(\frac{n \pi y^{\prime}}{b}\right) \\
G A_{y y 1}= & \frac{\mu}{4 a b} \sum_{m, n=0}^{\infty} \frac{\epsilon_{m} \epsilon_{n}}{k_{\rho_{m}, n}} f\left(\left|z-z^{\prime}\right|, k_{\rho_{m, n}}, E\right) \\
& \times \sin \left(\frac{m \pi x}{b}\right) \sin \left(\frac{m \pi x^{\prime}}{a}\right) \\
& \times \cos \left(\frac{n \pi y}{b}\right) \cos \left(\frac{n \pi y^{\prime}}{b}\right) \\
G A_{z z 1}= & \frac{\mu}{4 a b} \sum_{m, n=0}^{\infty} \frac{\epsilon_{m} \epsilon_{n}}{k_{\rho_{m, n}}} f\left(\left|z-z^{\prime}\right|, k_{\rho_{m, n}}, E\right) \\
& \times \sin \left(\frac{m \pi x}{b}\right) \sin \left(\frac{m \pi x^{\prime}}{a}\right) \\
& \times \sin \left(\frac{n \pi y}{b}\right) \sin \left(\frac{n \pi y^{\prime}}{b}\right) \\
G A_{\kappa \kappa}= & \frac{\mu}{4 \pi} \sum_{m, n=-\infty}^{\infty} \sum_{i=0}^{3} A_{i}^{\kappa \kappa} \frac{1}{R_{i, m n}} 2 \operatorname{Erfc}\left(R_{i, m n} E\right) \\
\kappa= & x, y, z
\end{aligned}
$$

It is remarkable to notice that, in above expressions, the (Erfc) functions in both terms will always contain real arguments, allowing faster calculations. Another interesting feature derived from the first fact is that the splitting parameter $(E)$ can always be chosen as the optimal one according to (8), so the summation of these series can be performed with very few terms. In fact, the imaginary part of the argument will never grow, as this imaginary part is always zero. Therefore, no numerical problems will be encountered during the evaluation of these complementary error functions.

Once the static term is evaluated in a very efficient way, the question that remains open is if the dynamic term in (12) can be further accelerated. The answer to this question is that the summation by parts algorithm presented in [10] can still be used for this term, when the observation point is not close to the source point. The formulation presented in [10] can be easily adapted to our dynamic series. First, we write equation (12) in the following form:

$$
\begin{aligned}
G A_{z z}^{\text {dynamic }}= & \frac{\mu}{2 a b} \sum_{m, n=0}^{\infty}\left(V_{m, n}-V_{m, n}^{a s y}\right) \\
& \times \sin \left(\frac{m \pi x}{b}\right) \sin \left(\frac{m \pi x^{\prime}}{a}\right) \\
& \times \sin \left(\frac{n \pi y}{b}\right) \sin \left(\frac{n \pi y^{\prime}}{b}\right) \\
= & \frac{\mu}{2 a b} \sum_{m=0}^{\infty} \sum_{n=0}^{\infty} \Upsilon(m, n) \\
& \times \sin \left(\frac{m \pi x}{b}\right) \sin \left(\frac{m \pi x^{\prime}}{a}\right) \\
& \times \sin \left(\frac{n \pi y}{b}\right) \sin \left(\frac{n \pi y^{\prime}}{b}\right) \\
= & \frac{\mu}{2 a b} \sum_{m=0}^{\infty} \sin \left(\frac{m \pi x}{b}\right) \sin \left(\frac{m \pi x^{\prime}}{a}\right) \\
& \times \sum_{n=0}^{\infty} \Upsilon(m, n) \sin \left(\frac{n \pi y}{b}\right) \sin \left(\frac{n \pi y^{\prime}}{b}\right)
\end{aligned}
$$

where we have made the redefinition

$$
\Upsilon(m, n)=\left(V_{m, n}-V_{m, n}^{a s y}\right) .
$$

For each value $\mathbf{m}$ of the external sum in (14), we can consider the infinite inner sum

$$
S_{\infty}^{\text {inner }}=\sum_{n=0}^{\infty} \Upsilon(\mathbf{m}, n) \sin \left(\frac{n \pi y}{b}\right) \sin \left(\frac{n \pi y^{\prime}}{b}\right)
$$

which, applying basic trigonometric relations, can be expressed as

$$
\begin{aligned}
S_{\infty}^{\text {inner }} & =\sum_{n=0}^{\infty} \Upsilon(\mathbf{m}, n) \\
& \times \frac{1}{2}\left[\cos \left(\frac{n \pi\left(y-y^{\prime}\right)}{b}\right)-\cos \left(\frac{n \pi\left(y+y^{\prime}\right)}{b}\right)\right] .
\end{aligned}
$$

Next, we will apply the summation by parts procedure as derived in [10] to this inner sum. As shown in [10], the procedure starts by splitting the sum into two terms

$$
S_{\infty}^{\text {inner }}=S_{N-1}^{\text {inner }}+R_{N}^{\text {inner }} .
$$

In (18), $S_{N-1}^{\text {inner }}$ is the partial sum from $(n=0)$ to $(N-1)$, and $R_{N}^{\text {inner }}$ is called the remainder, which can be transformed into [10]

$$
\begin{aligned}
R_{N}^{\text {inner }} & =\sum_{i=1}^{\infty} G_{N}^{(-i)} \\
\times & {\left[f_{N-1}^{(i+1)}\left(\frac{\left(y-y^{\prime}\right) \pi}{b}\right)-f_{N-1}^{(i+1)}\left(\frac{\left(y+y^{\prime}\right) \pi}{b}\right)\right] . }
\end{aligned}
$$


The index $(i)$ in (19) indicates the order of the summation by parts algorithm, $G_{N}^{(-i)}$ are partial differences of the original spectral domain function for $(n=N)$, and $f_{n}^{(i+1)}(x)$ are partial sums of the highly oscillating sinusoidal terms. Using the results presented in [10] we can write

$$
\begin{aligned}
G_{n}^{(-1)} & =\frac{\Upsilon(\mathbf{m}, n)}{2} ; \quad G_{n}^{(-i)}=G_{n+1}^{(-i+1)}-G_{n}^{(-i+1)} \\
f_{n}(x) & =\cos (n x) \\
f_{n}^{(+i)}(x) & =\sum_{k=n+1}^{\infty} f_{k}^{(i-1)}(x) ; \quad \text { for } i=2,3,4 \ldots
\end{aligned}
$$

The use of analytical closed form expressions for the partial sums $f_{n}^{(i)}(x)$ inside the brackets in (19), assures a rapid convergence of the remainder $R_{N}^{\text {inner }}$ (see [10, Eq. 8]). Thus, we can see that the expression of the total inner series (18) will also be accelerated, as the remaining term $S_{N-1}^{\text {inner }}$ is a fixed finite sum.

Once the inner sum is efficiently evaluated, the external sum exhibits a fast convergence behavior, and a direct evaluation is proposed. A similar procedure can be easily applied to the other components of the Green's functions. Only, when we have the product of cosine terms in the inner sum, then the summation of two cosine functions appears in (17).

It is important to notice that this procedure works well when the observation point is not close to the source point. In particular, [10, Eq. 8] completely fails if $y=y^{\prime}$. In this case we can follow an alternative strategy. If the difference of $x$ coordinates is large enough, we can still apply the technique by interchanging the inner and outer series. On the contrary, if we are also in a region where $x \approx x^{\prime}$, then we will not be able to apply the summation by parts technique for this case. It is important to remark that this strategy represents an important difference with respect to the algorithm proposed in [9]. Using the new strategy, the summation by parts technique can be applied successfully to all observation points fulfilling either $\left(x \neq x^{\prime}\right)$ or $\left(y \neq y^{\prime}\right)$. In the singular situation $\left(x \approx x^{\prime}\right.$ and $\left.y \approx y^{\prime}\right)$, the extension of the Kummer technique using a Taylor expansion is proposed in Section II-C, in order to accelerate the dynamic series.

\section{Acceleration of the Dynamic Term}

In the analysis of practical microwave circuits in a given frequency bandwidth, what it is important is the fast calculation of the dynamic term of (12). This is because this is the term depending on the frequency, and therefore, the term that needs to be computed at each frequency point in the analysis. We then concentrate the efforts in the acceleration of this dynamic term.

To increase the efficiency in the evaluation of the dynamic term for the singular situation described above, we propose to extract higher order asymptotic terms, following the Kummer strategy. Applying the Taylor expansion to the propagation constant $\left(\gamma_{m, n}\right)$, an asymptotic expression with two terms can be obtained (see the Appendix)

$$
V_{m, n}^{a s y}=e^{-k_{\rho_{m, n}}\left|z-z^{\prime}\right|}\left(\frac{1}{k_{\rho_{m, n}}}+\frac{1}{2} \frac{k^{2}}{k_{\rho_{m, n}}^{3}}\right) .
$$

If we look closely to (21), we can notice that we have obtained an expression consisting on the first asymptotic term we used previously, plus a second term exhibiting a $1 / k_{\rho_{m, n}}^{3}$ dependence. The use of a new asymptotic term in the Kummer technique allows to improve the convergence rate of the dynamic series, as it will be shown in the results section.

As a new asymptotic term has been subtracted from the original series, a second static sum must be added to recover the final Green's functions. The new static series adopt the following form:

$$
\begin{aligned}
S_{2} & =\frac{\mu}{2 a b} \sum_{m, n=0}^{\infty} \frac{1}{2} \frac{1}{k_{\rho_{m, n}}^{3}} e^{-k_{\rho_{m}, n}\left|z-z^{\prime}\right|} \\
\times & \sin \left(\frac{m \pi x}{a}\right) \sin \left(\frac{m \pi x^{\prime}}{a}\right) \sin \left(\frac{n \pi y}{b}\right) \sin \left(\frac{n \pi y^{\prime}}{b}\right) .
\end{aligned}
$$

It is important to notice that this series exhibits a good convergence behavior of the type $1 / k_{\rho_{m, n}}^{3}$, even in the critical case of $\left(z=z^{\prime}\right)$. Therefore, a direct evaluation of the series is possible. In addition, due to the static nature of the series, it has to be computed only once for a given geometry. Then, it does not add up new computational cost during the analysis of practical circuits in a given bandwidth.

Apart from these considerations, we can also accelerate the second asymptotic term by using the summation by parts technique, for observation points far from the source. Taking into account all these results, we can suggest a new strategy for computing efficiently the Green's functions for any combination of source/observer positions.

- First, if the difference $\left|z-z^{\prime}\right|$ is large enough, the spectral approach should be used, as it needs few modes to converge due to the intervening exponential term in (2a). Since this series involves only the evaluation of sinusoidal terms, it leads to faster calculations than the other forms of the series.

- On the contrary, if ( $\left.z \simeq z^{\prime}\right)$, we can apply the decomposition in the dynamic and static series, employing two asymptotic terms. For the first static term, the Ewald method is used to accelerate the series. For the dynamic series and for the second static term, we have to consider two more situations.

- If $\left(x \simeq x^{\prime}\right)$ and $\left(y \simeq y^{\prime}\right)$, the dynamic series and the second static series are computed directly in the spectral domain.

- In any other case, the two series can be efficiently evaluated with the summation by parts technique, employing very few terms as in the Ewald case.

For solving a MoM problem, the proposed approach allows to compute the whole MoM matrix as

$$
Z=Z_{\text {dynamic }}+Z_{\text {static } 1}+k^{2} Z_{\text {static } 2} ; \quad k=\frac{2 \pi}{\lambda} .
$$

The elements $Z_{\text {static1 }}$ and $Z_{\text {static2 }}$ are computed only once for a given geometry. Only the matrix $Z_{\text {dynamic }}$ is computed for every frequency point. The final impedance MoM matrix $(Z)$ is 


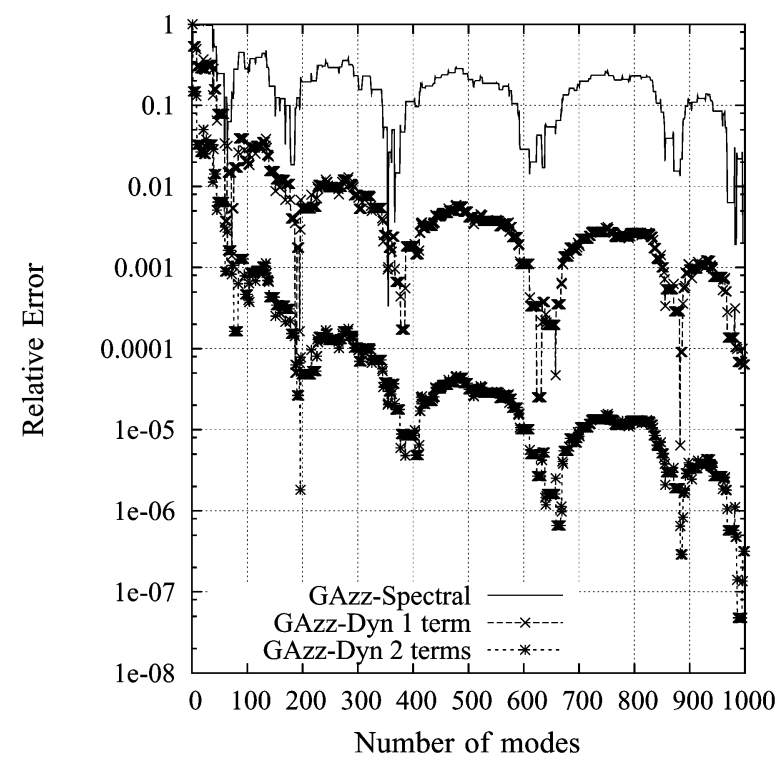

Fig. 2. Convergence rate for the total and dynamic series as a function of the number of modes, for the $G A_{z z}$ component. Similar behavior can be observed for the other components of the dyadic (not shown here).

recovered by introducing the frequency dependence as indicated in (23).

\section{RESULTS}

First, we are going to show the convergence rates for the different series formulated in the previous section. Let us consider the structure of Fig. 1 with $a=b=66 \mathrm{~mm}$. We will take the source position of Source 1 and the observation point $A$ (all the coordinates are shown in the addressed figure). For this test, the frequency is fixed to $15 \mathrm{GHz}$. In Fig. 2, the convergence rate for the $G A_{z z}$ component of the dyadic magnetic potential Green's function is presented for the critical case $\left(z=z^{\prime}\right)$. The figure presents the convergence of the original spectral series, and of the dynamic series when one and two Taylor terms are extracted. As explained in the previous section, the spectral series shows very poor convergence when $\left(z=z^{\prime}\right)$. On the contrary, the dynamic series shows better behavior, as a consequence of the subtraction of additional asymptotic terms. We observe from the figure that the extraction of the second static term allows to improve convergence, gaining about an order of magnitude in terms of precision for a fixed number of modes.

As the difference of $x$-coordinates between source and observation points is not very small for the given example, the summation by parts technique can be applied to further accelerate the dynamic series. The behavior of the summation by parts technique is good in this situation, as shown in Fig. 3.

First, Fig. 3(a) shows the convergence rate of the internal sum, where the summation by parts itself is performed. In this case convergence is very fast, obtaining relative errors better than $\left(10^{-6}\right)$ with an order of the reminder greater than $N=40$. The convergence behavior also shows that the relative error progressively reduces as the index $(i)$ grows [see (19)]. It is also interesting to observe that the precision is enhanced by increasing the order of the remainder $(N)$. When $(N)$ is too large, the numerical values of the successive differences in (20) are strongly

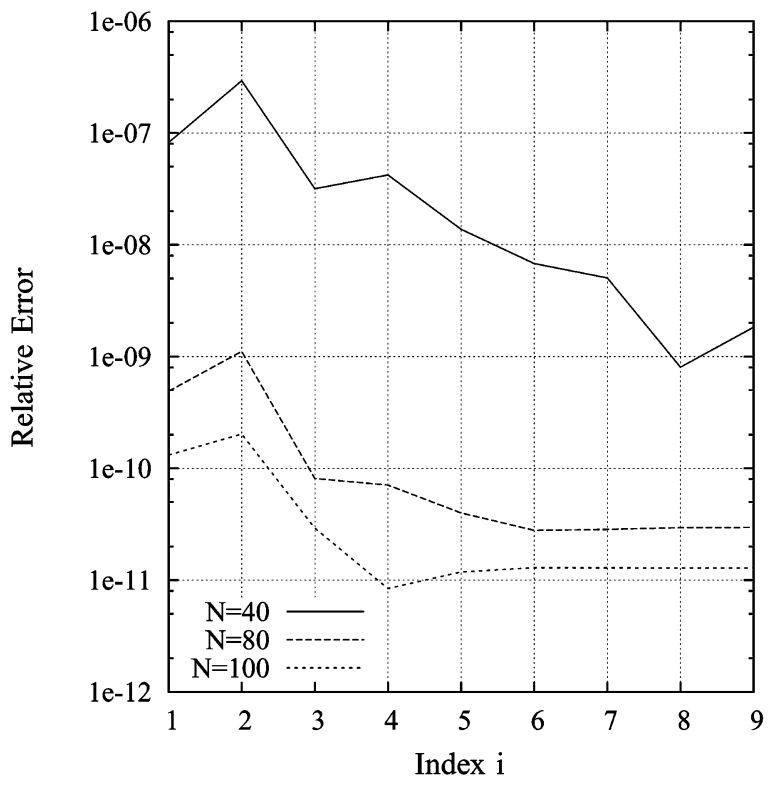

(a) Relative error of the internal sum versus the iteration index $(i)$ (see (19)), considering different values of the order of the remainder $(N)$.

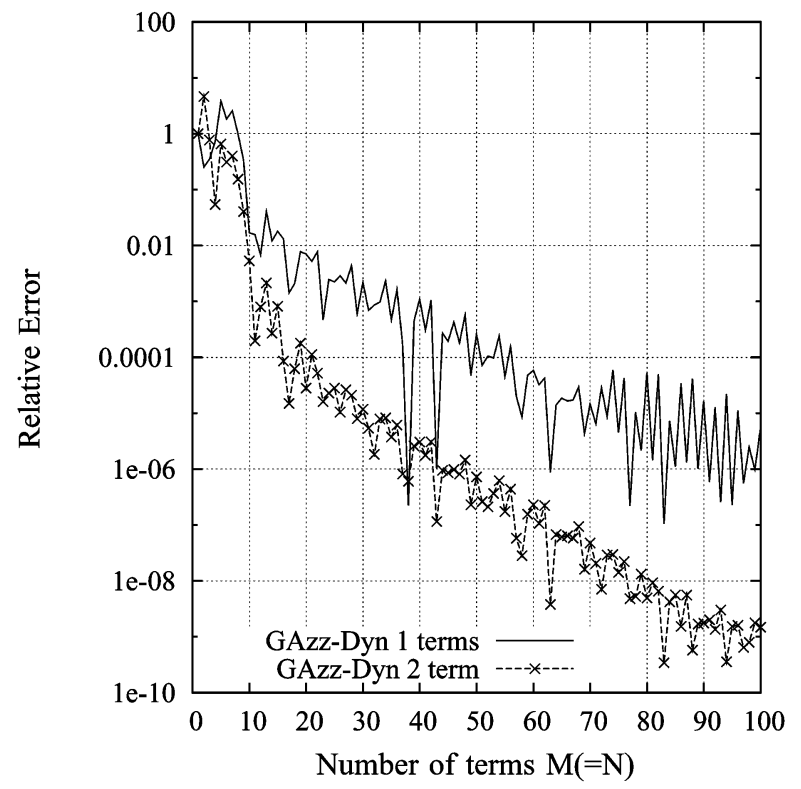

(b) Accelerated external sum, considering 1 and 2 asymptotic terms.

Fig. 3. Convergence rate for dynamic series of the $G A_{z z}$ component, accelerated with the summation by parts technique, for the same case as in Fig. 2. Similar behavior is observed for the other components of the dyadic (not shown here).

reduced as $(i)$ increases, leading to a saturation in the precision, as can be observed for the case of $N=100$. The important conclusion that arises from this result is that small values of $(i)$ and $(N)$ can be used to achieve acceptable error precisions better than $\left(10^{-4}\right)$.

On the other hand, Fig. 3(b) considers the external sum, which converges rapidly as a consequence of the acceleration of the previous inner series. In this case, the index $(i)$ has been fixed to the value of $(i=3)$, and the order of the remainder $(N)$ is taken of the same value as the number of terms $(M=N)$ used in the 


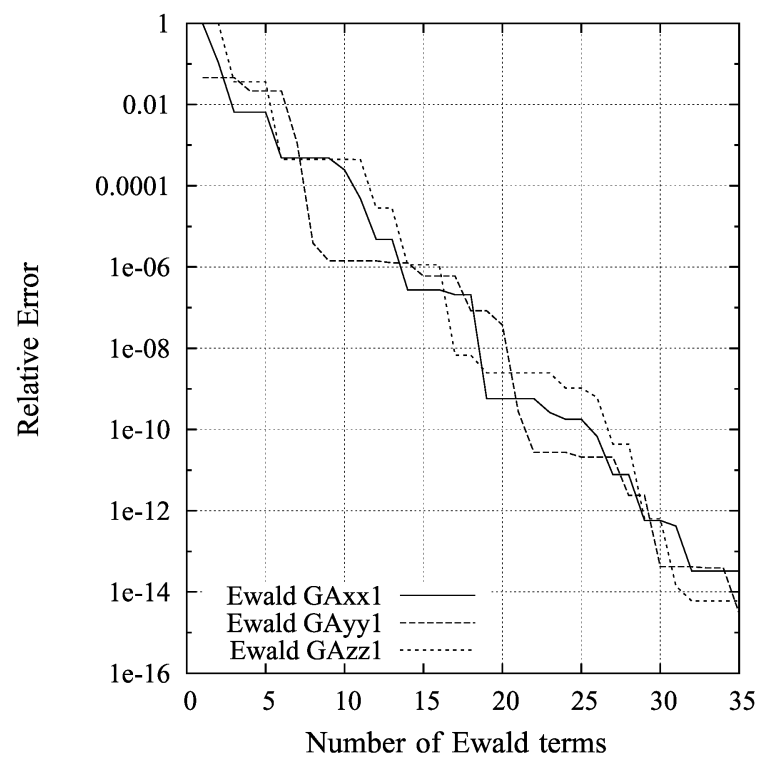

Fig. 4. Convergence rate for the first static series (using Ewald method), for the same case as in Fig. 2.

external sum. We can observe from the results that the convergence of the external sum is fast. In addition, the convergence rate for the external series is improved when a second Taylor term is extracted from the series. For example, for a fixed precision of $10^{-4}$, only 20 terms are needed when using two asymptotic terms, while 50 modes are needed if only one asymptotic term is extracted.

After studying the dynamic series, we will examine the static part, which must also be evaluated for the calculation of the Green's functions. The first static series, on which the Ewald method is applied, presents also a very fast convergence. Fig. 4 shows the relative error for the spectral components shown in (13a), as a function of the number of terms in the Ewald series. We can observe that just 10 Ewald terms are needed to achieve a precision of $10^{-4}$. The spatial components in (13d) converge even faster with the Ewald method, and only one term is needed to obtain the final value of the series (within the numerical precision of the machine), so they are not included here.

Next, let us study the convergence of the second static series. We have to bear in mind that the Ewald method cannot be applied to this series. Therefore, the same strategy as for the dynamic series will be used. Fig. 5(a) and (b) illustrates the convergence rates for the (22), considering both a direct evaluation and the summation by parts approaches. First, Fig. 5(a) shows that this series will converge even if a direct evaluation is applied. In addition, if the source point is far from the observation point, the summation by parts can be applied. In Fig. 5(b) the convergence rate of the external series is shown, when the internal series is summed with $(i=3)$ and $(N=40)$ in the summation by parts algorithm. The convergence behavior is similar to the one observed for the dynamic series. In particular, we obtain a relative error of $\left(10^{-4}\right)$ when 40 modes are used in the external series.

The only question remaining is how to combine efficiently the direct sum and the summation by parts, for the evaluation of the dynamic and of the second static term discussed above. To illustrate how this combination can be efficiently made, we present

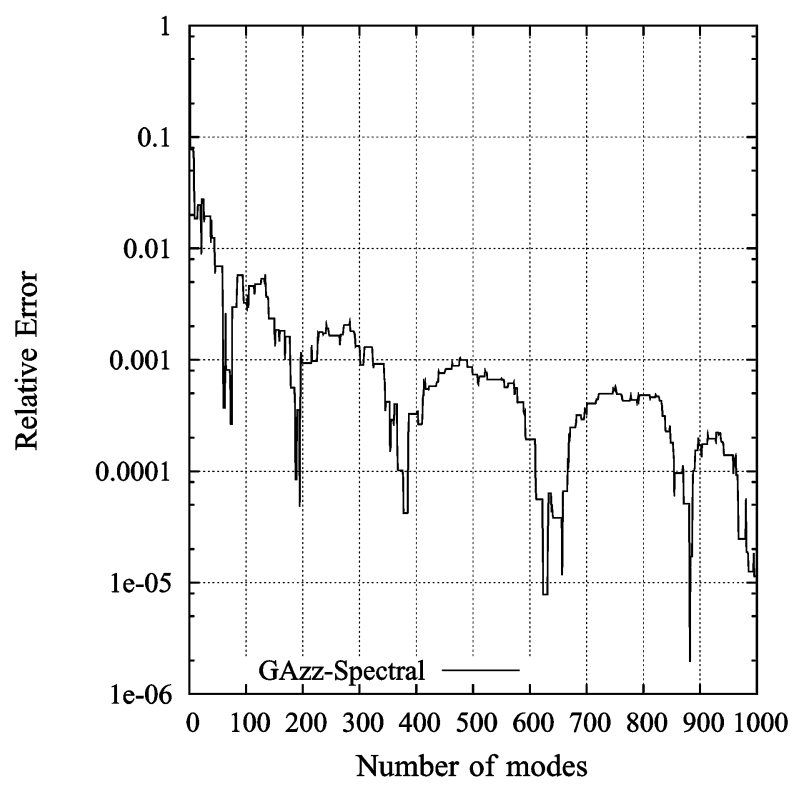

(a) Direct sum.

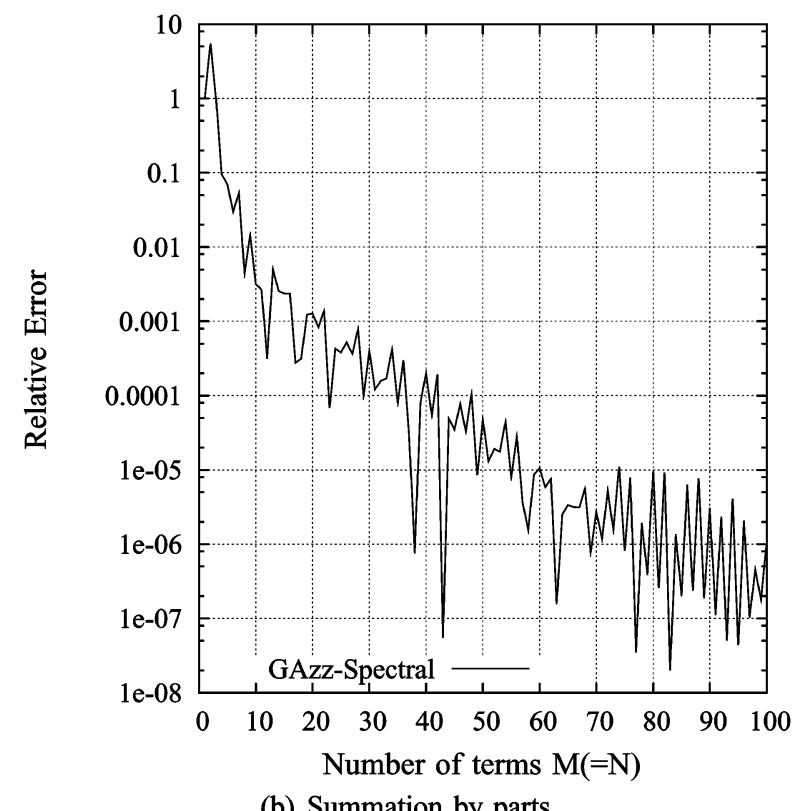

(b) Summation by parts.

Fig. 5. Convergence rate for the second static series, considering the same case as in Fig. 2.

in Fig. 6 the number of modes needed in the series to achieve a relative error of $10^{-4}$, when the observation point is varied along Line 2 shown in Fig. 1. In Fig. 6(a) we present the results obtained for the dynamic series when one and two asymptotic terms are extracted. We can observe that far from the singularity the curve is almost flat. This is because in this region the summation by parts is applied, and the precision is achieved with $13-15$ terms in the external series. This number of terms grows slightly as we get closer to the singularity. Once the observation point is inside the near-source region (when $x \approx x^{\prime}$ ), the direct sum is performed, and the number of modes needed to achieve the required precision increases. We see that very close to the source, the number of terms increases with a maximum of 750 modes. It is interesting to observe the important reduction in the number of modes required, when the second Taylor 


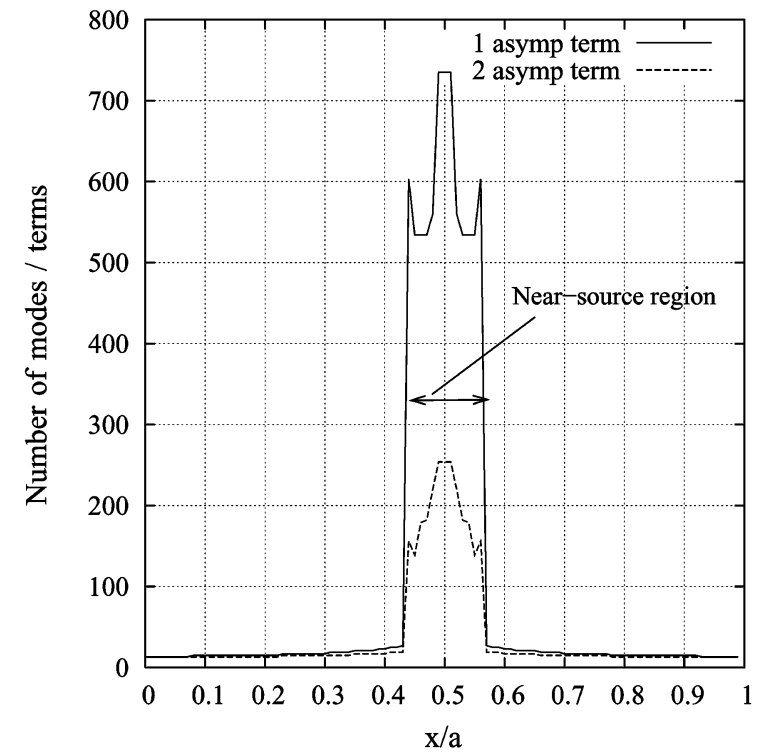

(a) Dynamic series, considering one and two asymptotic terms.

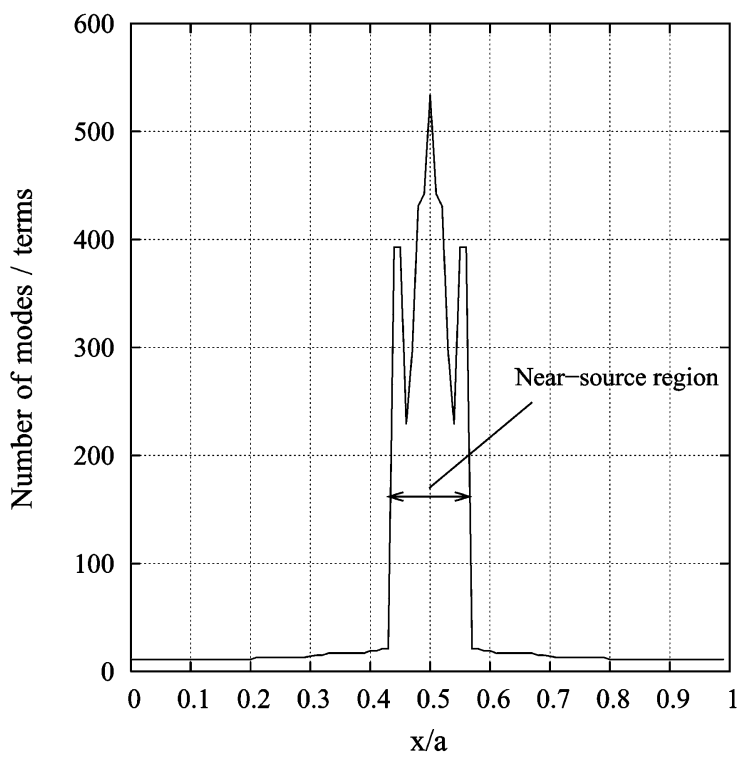

(b) Second asymptotic series.

Fig. 6. Number of terms needed to achieve a precision of $10^{-4}$ in the dynamic series when the observation point is varied along Line 2 of Fig. 1. The source point is placed at Source 1 of Fig. 1.

term is extracted from the series. The figure shows that only 250 modes are required to obtain the same precision, in the worst case. Moreover, far from the singularity, where the summation by parts is applied, very few modes are required in the external series to achieve the same precision as before.

A similar convergence study is presented in Fig. 6(b), but for the second asymptotic term [see (22)]. Far from the singularity, where the summation by parts is applied, only 11 terms are needed in the summation of the external series to achieve the required precision of $\left(10^{-4}\right)$. Close to the singularity the direct sum is applied. In this case we need 550 modes in the worst case to achieve the required precision. However, it is important to recall that this computation will have to be done only once during the analysis of a practical circuit in a given bandwidth.
TABLE I

COMPARISON BETWEEN OUR APPROACH AND THE EWALD METHOD FOR A 1000 POINTS FREQUENCY SWEEP

\begin{tabular}{|c|c|c|c|c|}
\hline $\begin{array}{c}\text { Waveguide } \\
\text { dimensions }\end{array}$ & Method & $E$ Value & $\begin{array}{c}\text { Number } \\
\text { Ewald } \\
\text { terms }\end{array}$ & $\begin{array}{c}\text { Time } \\
(\text { secs })\end{array}$ \\
\hline \hline $\begin{array}{c}a=21.86 \mathrm{~mm} \\
b=11.06 \mathrm{~mm}\end{array}$ & Ewald & 113.99 & 10 & 18.075 \\
\hline $\begin{array}{c}a=21.86 \mathrm{~mm} \\
b=11.06 \mathrm{~mm}\end{array}$ & $\begin{array}{c}\text { Dynamic } \\
\text { - Static }\end{array}$ & 113.99 & 10 & 3.875 \\
\hline $\begin{array}{c}a=66 \mathrm{~mm} \\
b=66 \mathrm{~mm}\end{array}$ & Ewald & 103.98 & 80 & 764.389 \\
\hline $\begin{array}{c}a=66 \mathrm{~mm} \\
b=66 \mathrm{~mm}\end{array}$ & $\begin{array}{c}\text { Dynamic } \\
\text { - Static }\end{array}$ & 23.8 & 10 & 3.855 \\
\hline
\end{tabular}

In the above analysis, the calculations of the Green's functions have been carried out using different techniques according to the singular condition of the observer point, as related to the source position. For an efficient calculation, it is important to define correctly the limits of the so called near-source region, where the direct sum is to be applied. If the near-source region is defined too small, the summation by parts will not be effective for observation points very close to the source. On the contrary, if the near-source region is selected too big, we will end up applying the direct sum for too many observation points, with the corresponding loss in efficiency. After a fine tuning of the summation by parts technique, we have found that the most efficient way to define the near-source region is for observation points satisfying the following conditions:

$$
\left[\frac{\pi\left(x-x^{\prime}\right)}{a} \leq \eta\right] ; \quad\left[\frac{\pi\left(y-y^{\prime}\right)}{b} \leq \eta\right]
$$

where $(\eta)$ is a threshold used to switch from the near-source region to the far region. We have seen that the best efficiency is obtained when this threshold takes a value around $\eta=0.2$. Apart from the better convergency rates, the most important feature of the proposed method is the independence with frequency of the static series. To show the importance of this fact,Table I compares the computational time between the normal Ewald approach used in [12] (complex argument case), and the dynamicstatic series decomposition with two asymptotic terms used in this paper. Computations were made on a $2.8 \mathrm{GHz}$ Pentium IV processor, using the same observation and source points in two different waveguides. The time reported in the table gives the CPU time needed for the evaluation of 1000 frequency points inside the band from $10 \mathrm{GHz}$ to $20 \mathrm{GHz}$, considering Source 1 and point $A$ from Fig. 1.

In the first waveguide, the value of the splitting parameter $(E)$ remains the same for both methods [applying (8)]. However, with the approach derived in this paper, the Ewald technique is applied only once to the first asymptotic term. This yields an important reduction in the computational time, as shown inTable I. The time difference is even larger for the case of the second bigger waveguide. In this case the splitting parameter $(E)$ must be increased in the direct Ewald approach [using (9)]. This slows down the convergence rate of the Ewald technique, requiring 80 terms in the series to reach the precision of $\left(10^{-4}\right)$. For the approach proposed in this paper, the splitting parameter is still selected with (8), since it is applied only to the static situation. This 
leads to a smaller value of the splitting parameter, and hence to a faster convergence. The gain in efficiency shown in Table $I$ is really important.

In order to validate the method proposed, we present in Fig. 7 the numerical results for the electric scalar potential Green's function $(G v)$, along lines 1 and 2 shown in Fig. 1. The waveguide dimensions are $(a=b=66 \mathrm{~mm})$, and we have considered two source positions (Source 1 and Source 2 shown in Fig. 1). Comparisons between the technique presented in this paper with a direct application of the spectral and the spatial approaches, are included. Also, results obtained with the technique developed in [9] for multilayered cavities are presented for validation. In the case of Fig. 7(a), the observer point is always far from the source point. Therefore, using our approach, the summation by parts technique is always used for the calculation of the Green's function (the observer point never enters into the near-source region). In fact, when $\left(x \approx x^{\prime}\right)$, the inner and outer series are interchanged, and the summation by parts is applied to the $y$-coordinate. This is an important advantage of the strategy proposed in this paper, as compared to the two dimensional summation by parts technique presented in [9]. We have observed that the precision of $\left(10^{-4}\right)$ is reached for all observation points with a reminder of $(N=20)$, using three summation by parts iterations $(i=3)$.

Finally, Fig. 7(b) shows a spatial sweep similar to the previous one, but using line 2 shown in Fig. 1. In this case the sweep goes close to the source, so the technique implemented in this paper will have to switch between the summation by parts technique and the direct sum of the dynamic series with two Taylor terms extracted. The validation of the technique with the results obtained using [9] shows that accuracy is maintained also for observation points very close to the source.

\section{CONCLUSION}

In this paper, we have presented a new approach for the efficient calculation of the Green's functions inside a rectangular waveguide. After exploring the conventional formulations (spectral and spatial domain series), a new strategy has been proposed. The idea is to apply the Kummer transformation, using two asymptotic terms of the spectral domain Green's functions, obtained through a Taylor series expansion. Instead of applying the Ewald method to the whole series, it is only applied to the first asymptotic series. In this way the number of terms needed in the Ewald technique is drastically reduced, avoiding the numerical instabilities associated to the proper selection of the splitting parameter. A key issue for the numerical efficiency of the technique, is that only the dynamic term of the series need to be recomputed for each new frequency point. We have demonstrated that the dynamic series is accelerated by an order of magnitude, by extracting the second Taylor term from the original series. In addition, efficiency is increased by using the summation by parts technique, when the source point is placed far from the observer point. A new strategy for the use of the summation by parts technique was presented in this paper. Following this approach, the summation by parts is only applied to the inner series. By interchanging the inner and outer series, we have extended the range of applicability of the summation by parts technique, as compared to previous

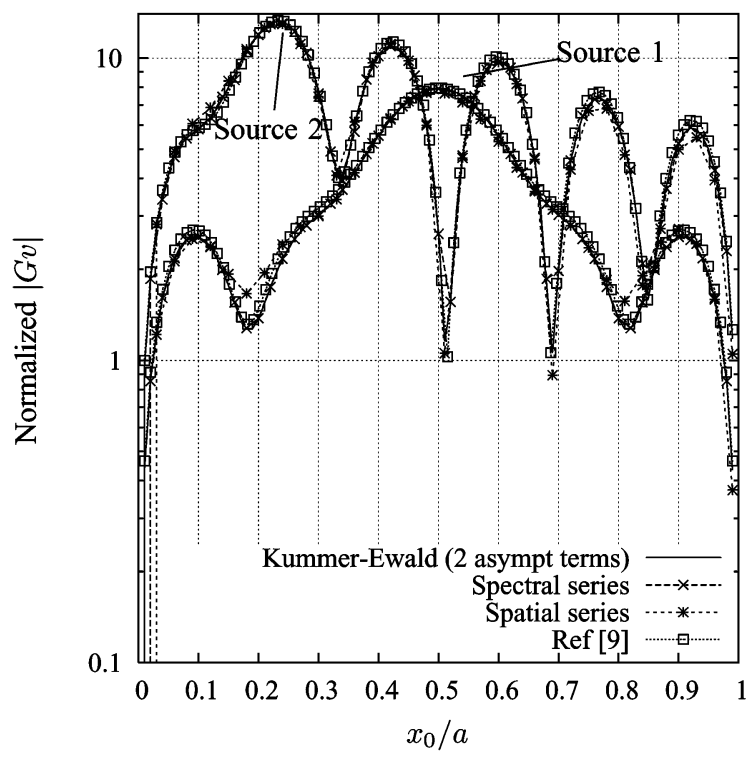

(a) Spatial sweep along line 1 .

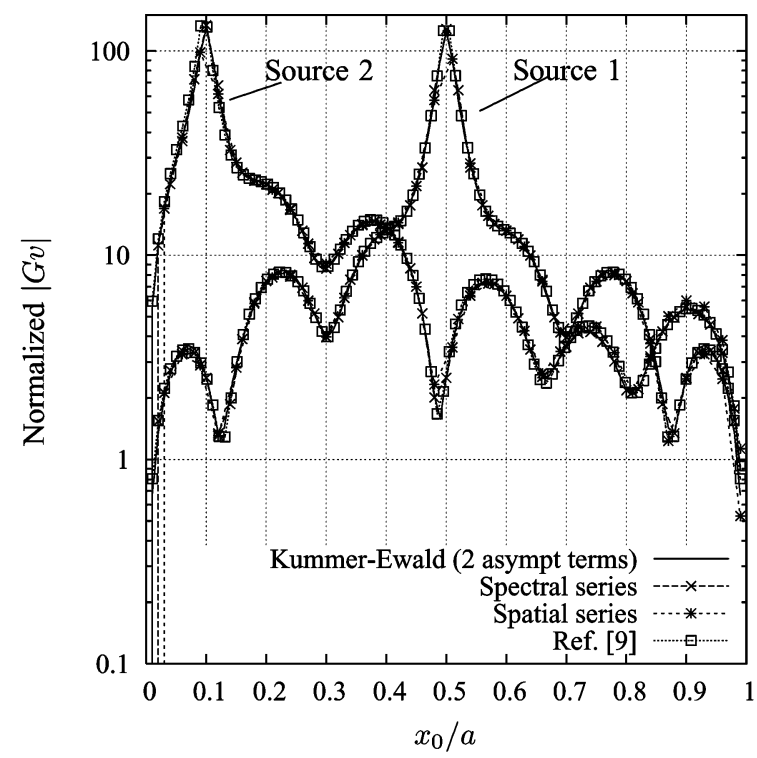

(b) Spatial sweep along line 2 .

Fig. 7. Electric scalar potential Green's function $(G v)$ when the observation point is varied along Line 1 and Line 2 of Fig. 1, when the source is placed at Source 1 and Source 2 also shown in Fig. 1. Frequency is $15 \mathrm{GHz}$.

works. The new developed Green's functions are validated with other techniques, showing that accuracy is preserved even for observation points very close to the source point.

\section{APPENDIX}

\section{TAYLOR EXPANSION OF THE ASYMPTOTIC EXPRESSION}

In this appendix we give the details of the Taylor expansion used to calculate the two asymptotic terms, needed in the Kummer transformation used in the paper.

To proceed, we will start by rewriting the expression of $\left(\gamma_{m, n}\right)$ as

$$
\gamma_{m, n}=\sqrt{k_{\rho_{m, n}}^{2}-k^{2}}=k_{\rho_{m, n}} \sqrt{1-\left(\frac{k}{k_{\rho_{m, n}}}\right)^{2}} .
$$


Now we can use the Taylor expansion of the function $\sqrt{1+x}$

$$
\sqrt{1+x}=1+\frac{1}{2} x-\frac{3}{8} x^{2} \ldots
$$

considering $x=-\left(k / k_{\rho_{m n}}\right)^{2}$, and taking the first two terms, we can apply (26) to (25), obtaining

$$
\gamma_{m, n} \approx k_{\rho_{m, n}}\left[1-\frac{1}{2}\left(\frac{k}{k_{\rho_{m, n}}}\right)^{2}\right]
$$

Employing the new expression for $\gamma_{m, n}$, a new asymptotic term for the spectral Green's functions can be obtained as

$$
V_{m, n}^{\mathrm{asymp}}=\frac{e^{-k_{\rho_{m, n}}\left|z-z^{\prime}\right|} e^{1 / 2 k^{2} / k_{\rho_{m, n}}}}{k_{\rho_{m, n}}\left[1-\frac{1}{2}\left(\frac{k}{k_{\rho_{m}, n}}\right)^{2}\right]} .
$$

Here, it is convenient to use another common Taylor expansion, to separate the above expression into simple terms

$$
\frac{1}{1-x} \approx 1+x
$$

Applying (29) to (28), the following expression is obtained:

$$
\begin{aligned}
V_{m, n}^{\operatorname{asymp}}= & \frac{e^{-k_{\rho_{m, n}}\left|z-z^{\prime}\right|} e^{1 / 2 k^{2} / k_{\rho_{m, n}}\left|z-z^{\prime}\right|}}{k_{\rho_{m, n}}} \\
& \times\left[1+\frac{1}{2}\left(\frac{k}{k_{\rho_{m, n}}}\right)^{2}\right] \\
= & e^{-k_{\rho_{m, n}}\left|z-z^{\prime}\right|} e^{1 / 2 k^{2} / k_{\rho_{m, n}}\left|z-z^{\prime}\right|} \\
& \times\left(\frac{1}{k_{\rho_{m, n}}}+\frac{1}{2} \frac{k^{2}}{k_{\rho_{m, n}}^{3}}\right) .
\end{aligned}
$$

Next, we may use a new Taylor expansion, this time for the exponential function

$$
e^{x} \approx 1+x
$$

Using (31) on the second exponential in (30), we obtain

$$
\begin{aligned}
V_{m, n}^{\text {asymp }}=e^{-k_{\rho_{m, n}}\left|z-z^{\prime}\right|}(1+ & \left.\frac{1}{2} \frac{k^{2}}{k_{\rho_{m n}}}\left|z-z^{\prime}\right|\right) \\
& \times\left(\frac{1}{k_{\rho_{m, n}}}+\frac{1}{2} \frac{k^{2}}{k_{\rho_{m, n}}^{3}}\right) .
\end{aligned}
$$

We finally expand this expression, and assuming $k_{\rho_{m, n}} \gg$ $k^{2}$, we retain only the first two terms in the expansion, obtaining finally

$$
V_{m, n}^{\text {asymp }}=e^{-k_{\rho_{m, n}}\left|z-z^{\prime}\right|}\left(\frac{1}{k_{\rho_{m, n}}}+\frac{1}{2} \frac{k^{2}}{k_{\rho_{m, n}}^{3}}\right) .
$$

\section{REFERENCES}

[1] I. Stevanovic, P. Crespo-Valero, K. Blagovic, and J. R. Mosig, "Integral-equation analysis of 3-D metallic objects arranged in 2-D lattices using the Ewald transformation," IEEE Trans. Microw. Theory Tech., vol. 54, pp. 3688-3697, Oct. 2006.

[2] G. Valerio, P. Baccarelli, P. Burghignoli, and A. Galli, "Comparative analysis of acceleration techniques for 2-D and 3-D Green's functions in periodic structures along one and two directions," IEEE Trans. Microw. Theory Tech., vol. 55, pp. 1630-1643, Jun. 2007.

[3] S. Singh, W. F. Richards, J. R. Zinecker, and D. R. Wilton, "Accelerating the convergence of series representing the free space periodic Green's functions," IEEE Trans. Antennas Propag., vol. 38, pp. 1958-1962, Dec. 1990.

[4] G. G. Gentili, L. E. Garcia-Castillo, M. Salazar-Palma, and F. PerezMartinez, "Green's function analysis of single and stacked rectangular microstrip patch antennas enclosed in a cavity," IEEE Trans. Antennas Propag., vol. 45, pp. 573-579, Apr. 1997.

[5] G. V. Eleftheriades, J. R. Mosig, and M. Guglielmi, "A fast integral equation technique for shielded planar circuits defined on nonuniform meshes," IEEE Trans. Microw. Theory Tech., vol. 44, pp. 2293-2296, Dec. 1996.

[6] T. F. Eibert, J. L. Volakis, D. R. Wilton, and D. R. Jackson, "Hybrid FE/BI modeling of 3-D doubly periodic structures utilizing triangular prismatic elements and an MPIE formulation accelerated by the Ewald transformation," IEEE Trans. Antennas Propag., vol. 47, pp. 843-850, May 1999.

[7] J.-Y. Lee, T.-S. Horng, and N. G. Alexopoulos, "Analysis of cavitybacked aperture antennas with a dielectric overlay," IEEE Trans. Antennas Propag., vol. 42, pp. 1556-1562, Nov. 1994.

[8] K. A. Michalski and J. R. Mosig, "Multilayered media Green's functions in integral equation formulations," IEEE Trans. Antennas Propag., vol. 45, pp. 508-519, Mar. 1997.

[9] A. A. Melcon and J. R. Mosig, "Two techniques for the efficient numerical calculation of the Green's functions for planar shielded circuits and antennas," IEEE Trans. Microw. Theory Tech., vol. 48, pp. 1492-1504, Sep. 2000.

[10] J. R. Mosig and A. A. Melcon, "The summation by parts algorithm: A new efficient technique for the rapid calculation of certain series arising in shielded planar structures," IEEE Trans. Microw. Theory Tech., vol. 50, pp. 215-218, Jan. 2002.

[11] M.-J. Park and S. Nam, "Rapid calculation of the Green's function in the shielded planar structures," IEEE Microw. Guided Wave Lett., vol. 7, pp. 326-328, Oct. 1997.

[12] M.-J. Park and S. Nam, "Rapid summation of the Green's function for the rectangular waveguide," IEEE Trans. Microw. Theory Tech., vol. 46, pp. 2164-2166, Dec. 1998.

[13] F. Capolino, D. R. Wilton, and W. A. Johnson, "Efficient computation of the 2-D Green's function for 1-D periodic structures using the Ewald method," IEEE Trans. Antennas Propag., vol. 53, pp. 2977-2984, Sep. 2005.

[14] A. Kustepeli and A. Q. Martin, "On the splitting parameter in the Ewald method," IEEE Trans. Microw. Guided Wave Lett., vol. 10, pp. 168-171, May 2000.

[15] P. P. Ewald, "Die berechnung optischer und elektrostatischen gitterpotentiale," Ann. Phys., vol. 64, pp. 253-268, 1921.

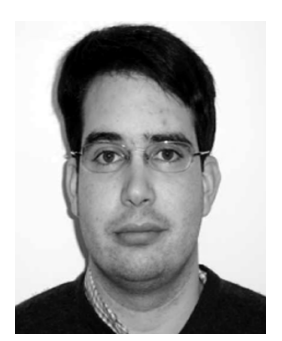

Francisco Javier Perez Soler (S'05) was born in Murcia, Spain, on January 16, 1981. He received the Electrical Engineering degree from the Technical University of Cartagena, Cartagena, Spain, in 2004, where he is currently working toward the Ph.D. degree.

Since 2007, he has been working as an Assistant Professor in the Communications and Information Technologies Department, Technical University of Cartagena. His current research interests include the areas of integral equation methods and fast numerical techniques for analysis of microwave components, waveguides and cavity structures including dielectric objects. 


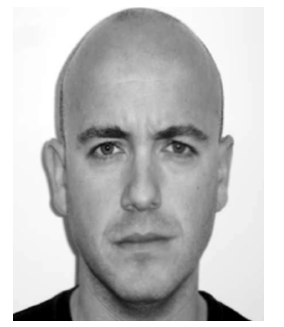

Fenando D. Quesada Pereira (S'05-M'07) was born in Murcia, Spain, in 1974. He received the Telecommunications Engineer degree from the Technical University of Valencia (UPV), Valencia, Spain, in 2000, and the Ph.D. degree from the Technical University of Cartagena (UPCT), Cartagena, Spain in 2007.

In 1999, he joined the Radiocommunications Department, UPV, as a Research Assistant, where he was involved in the development of numerical methods for the analysis of anechoic chambers and tag antennas. In 2001, he joined the Communications and Information Technologies Department, UPCT, initially as a Research Assistant, and then as an Assistant Professor. In 2005, he spent six months as a Visiting Scientist with the University of Pavia, Pavia, Italy. His current scientific interests include IE numerical methods for the analysis of antennas and microwave devices.

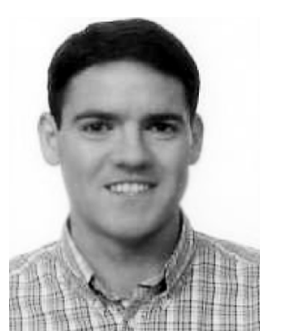

David Cañete Rebenaque (S'04) was born in Valencia, Spain, in 1976. He received the Telecommunications Engineer degree from the Technical University of Valencia (UPV), Valencia, Spain, in 2000 , and is currently working toward the Ph.D. degree at the Technical University of Cartagena (UPCT), Cartagena, Spain.

During 2001, he was an RF Engineer with a mobile communications company. In 2002, he joined the Communications and Information Technologies Department, UPCT, initially as a Research Assistant, and then as an Assistant Professor. His research interests include the analysis and design of microwave circuits and active antennas.

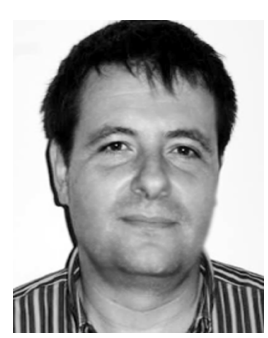

Alejandro Alvarez Melcon (M'99) was born in Madrid, Spain, in 1965. He received the Telecommunications Engineer degree from the Technical University of Madrid (UPM), Madrid, Spain, in 1991, and the Ph.D. degree in electrical engineering from the Swiss Federal Institute of Technology, Lausanne, Switzerland, in 1998.

In 1988, he joined the Signal, Systems and Radiocommunications Department, UPM, as a Research Student, where he was involved in the design, testing, and measurement of broadband spiral antennas for electromagnetic measurements support (EMS) equipment. From 1991 to 1993 , he was with the Radio Frequency Systems Division, European Space Agency (ESA/ESTEC), Noordwijk, The Netherlands, where he was involved in the development of analytical and numerical tools for the study of waveguide discontinuities, planar transmission lines, and microwave filters. From 1993 to 1995, he was with the Space Division, Industry Alcatel Espacio, Madrid, Spain, and was also with the ESA, where he collaborated in several ESA/European Space Research and Technology Centre (ESTEC) contracts. From 1995 to 1999, he was with the Swiss Federal Institute of Technology, Ecole Polytechnique Fédérale de Lausanne (EPFL), Lausanne, Switzerland, where he was involved with the field of microstrip antennas and printed circuits for space applications. In 2000, he joined the Technical University of Cartagena, Cartagena, Spain, where he is currently developing his teaching and research activities.

Dr. Melcon was the recipient of the Journe Internationales de Nice Sur les Antennes (JINA) Best Paper Award for the best contribution to the JINA'98 International Symposium on Antennas, and the Colegio Oficial de Ingenieros de Telecomunicacin (COIT/AEIT) Award to the best Ph.D. thesis in basic information and communication technologies.

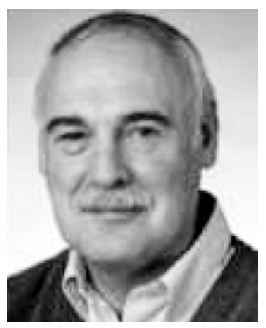

Juan R. Mosig (S'76-M'87-SM'94-F'99) was born in Cadiz, Spain. He received the Electrical Engineer degree from the Universidad Politécnica de Madrid, Madrid, Spain, in 1973, and the Ph.D. degree from the Ecole Polytechnique Fédérale de Lausanne (EPFL), Lausanne, Switzerland, in 1983.

In 1976, he joined the Laboratory of Electromagnetics and Acoustics, EPFL. Since 1991, he has been a Professor with EPFL, and since 2000, he has been the Head of the Laboratory of Electromagnetics and Acoustics (LEMA). In 1984, he was a Visiting Research Associate with the Rochester Institute of Technology, Rochester,NY, and Syracuse University, Syracuse, NY. He has also held scientific appointments with the University of Rennes, Rennes, France, the University of Nice, Nice, France, the Technical University of Denmark, Lyngby, Denmark, and the University of Colorado at Boulder. He is co-organizer and lecturer of yearly short courses in numerical electromagnetics in both Europe and the U.S. He is also responsible for several research projects of the European Space Agency (ESA). He has authored four chapters in books on microstrip antennas and circuits and over 100 reviewed papers. His research interests include EM theory, numerical methods, and planar antennas.

Dr. Mosig is a member of the Swiss Federal Commission for Space Applications and a member of the Executive Board of the European Network of Excellence ACE (2004-2007). He is the Chairman of the European COST Project on Antennas (2003-2006). 